\title{
Angle constraint active management of distribution networks with wind power
}

DOI:

10.1109/ISGTEUROPE.2010.5638966

Link to publication record in Manchester Research Explorer

\section{Citation for published version (APA):}

Ochoa, L. F., \& Wilson, D. H. (2010). Angle constraint active management of distribution networks with wind power. In IEEE PES Innovative Smart Grid Technologies Conference Europe, ISGT Europe/IEEE PES Conf. Innov. Smart Grid Technol. Eur., ISGT Eur. https://doi.org/10.1109/ISGTEUROPE.2010.5638966

\section{Published in:}

IEEE PES Innovative Smart Grid Technologies Conference Europe, ISGT Europe|IEEE PES Conf. Innov. Smart Grid Technol. Eur., ISGT Eur.

\section{Citing this paper}

Please note that where the full-text provided on Manchester Research Explorer is the Author Accepted Manuscript or Proof version this may differ from the final Published version. If citing, it is advised that you check and use the publisher's definitive version.

\section{General rights}

Copyright and moral rights for the publications made accessible in the Research Explorer are retained by the authors and/or other copyright owners and it is a condition of accessing publications that users recognise and abide by the legal requirements associated with these rights.

\section{Takedown policy}

If you believe that this document breaches copyright please refer to the University of Manchester's Takedown Procedures [http://man.ac.uk/04Y6Bo] or contact uml.scholarlycommunications@manchester.ac.uk providing relevant details, so we can investigate your claim.

\section{OPEN ACCESS}




\title{
Angle Constraint Active Management of Distribution Networks with Wind Power
}

\author{
Luis F. Ochoa, Member, IEEE, and Douglas H. Wilson
}

\begin{abstract}
Large volumes of wind generation capacity are expected to connect to distribution networks in the coming years. The traditional 'fit and forget' approach where all technical limitations are satisfied in any credible operational scenario has significantly reduced the ability of certain networks to integrate more generation capacity as the extra costs are not viable for most wind farm developers. In this context, it is envisaged that the true potential of distribution networks to accommodate large renewable generation capacities will only be realised by applying active network management schemes. Here, it is proposed the innovative use of synchrophasor technology to actively manage wind power generation output in congested distribution networks, resulting in the connection of more capacity and, hence, the delivery of more energy as opposed to the 'fit and forget' approach. This is achieved by applying an angle-based constraint that is determined according to the network characteristics (i.e., a proxy for thermal limits, voltage limits, etc.) and using minimal communication. Results from a radial test feeder considering two wind farms demonstrate the effectiveness of the technique in exporting more energy, although at expense of smaller capacity factors, whilst keeping the system secure.
\end{abstract}

Index Terms-Distributed generation, active management, smart grids, PMUs, wind power, optimal power flow, distribution networks.

\section{INTRODUCTION}

$\mathrm{E}^{\mathrm{s}}$ NVIRONMENTAL and security of supply concerns have placed investment in low-carbon power generation technologies as one of the priorities on the energy agendas of many countries around the world. We expect a vast acceleration in the connection of renewables over the next decade, primarily in the form of wind power, to both transmission and distribution networks. In the UK alone, $10 \mathrm{GW}$ of additional distributed generation (DG) capacity have been forecast by Distribution Network Operators (DNOs) to connect in the period of 2010-2015 [1]. However, present distribution networks, traditionally designed to be passive circuits with unidirectional power flows, are quickly becoming one of the major bottlenecks for the future deployment of DG.

Current practice in the UK is to provide DG operators with a firm connection. Such an approach, also known as 'fit and forget', requires that all technical limitations such as voltage

L. F. Ochoa is with Psymetrix Limited, Edinburgh, EH6 7BD, U.K. and the Institute for Energy Systems, School of Engineering, University of Edinburgh, Edinburgh, EH9 3JL, U.K. (e-mail: luis_ochoa@ieee.org)

D. H. Wilson is with Psymetrix Limited, Edinburgh, EH6 7BD, U.K. (email: douglas.wilson@psymetrix.com) rise, thermal ratings and avoidance of reverse power flows through grid transformers (if necessary) are satisfied in any credible operational scenario (e.g., minimum demandmaximum generation, $\mathrm{N}-1$ or $\mathrm{N}-2$ contingencies, etc.). While this might be considered a secure, reliable and proven approach, it requires expensive traditional reinforcements and neglects the fact that renewable sources, such as wind power, are inherently variable and hence there will be many periods where assets are not fully utilised. Indeed, these fit-and-forget connections have significantly curtailed the ability of certain networks to integrate more generation capacity as the extra costs are not viable for most DG developers. Although alternative connections based on intertripping schemes that actuate upon the occurrence of a constraint breach or a contingency, known as non-firm connections, allow the integration of more DG capacity, the true potential of distribution networks to accommodate large generation capacities by applying active management has yet to be realised.

Active Network Management (ANM), i.e., the use of realtime control and communication systems to better integrate and exploit the different network assets and participants, can be considered as a building block of the envisaged Smart Distribution Networks. Nonetheless, despite the potential benefits of ANM on the accommodation of larger volumes of DG capacity [2-4], DNOs have not yet widely adopted this 'connect and manage' approach mainly due to regulatory and commercial barriers. This is expected to change in the near future as governments promote innovation through funding and regulatory mechanisms (e.g., The Recovery Act in USA, and the Ofgem Low Carbon Network Fund in UK).

This work demonstrates the innovative use of synchrophasor measurement technology [5], a technology that has been widely deployed in transmission systems over the last decade for measurement, analysis and control of stability, and is able to acquire data at a fast speed (up to $60 \mathrm{~Hz}$ ) with very precise GPS time synchronisation. Here, it is proposed that synchrophasor measurements are used to actively manage wind power generation output in congested distribution networks, resulting in the connection of more capacity and, hence, the delivery of more energy as opposed to the fit-andforget approach. This is achieved by applying an angle-based constraint that is determined according to the network characteristics (i.e., a proxy for thermal and voltage limits, avoidance of reverse power flow through grid transformers, etc.) and using minimal communication. A patent for this technique has recently been applied for (UK Patent App. No.: 


\section{Accepted Paper}

\section{GB0920206.0).}

This paper is laid out as follows: Section II presents how synchrophasor measurements can capture demand and generation behaviour in distribution networks. In section III, a radial test feeder is used to demonstrate the Angle Constraint Active Management (ACAM) methodology. A previously developed ac Optimal Power Flow (OPF)-based algorithm is used to obtain the optimal setting for the angle constraint. Real time-series demand and wind speed data are used to determine the performance of the ACAM scheme. Section IV discusses practical issues on the implementation of the proposed approach. Conclusions are drawn in section V.

\section{Phase Angles AND Distribution Networks}

In power systems, the magnitudes of voltages and currents (or power flows) are probably the most important parameters to be measured and/or estimated for steady state analyses. Due to the established phasor nature of voltages and currents (and derived parameters), their corresponding phase angles need also to be incorporated into the ac models of transmission and distribution networks. However, the actual applicability or usefulness of monitoring and understanding the behaviour of these phase angles has only been demonstrated at transmission levels, particularly for the analysis and control of stability [6], and to some extent islanding. As for distribution networks, traditionally designed as passive circuits, stability issues are usually negligible or simply do not exist - although this scenario might change with large volumes of distributed generation being connected in the next decade. In general, the only application of synchrophasor measurements seen by distribution networks has been model validation.

Similar to the voltage magnitude, the corresponding phase angle is able to capture the different demand levels of a network. Adopting the test feeder shown in Fig. 1 and assuming a non-DG scenario, Fig. 2 presents the voltage magnitudes and corresponding phase angles (GSP taken as the reference bus) for different levels of total demand (from low, $3 \mathrm{MW}$, to peak, 10MW). Although transformer and line impedances have an impact on the resulting phase angle $(\mathrm{V}=\mathrm{IZ}$ ), with the same network configuration it is clear that the variability of the demand can be captured by synchrophasor measurements at any point of the feeder: the larger the demand, the lower (i.e., more negative) the value of the phase angle.

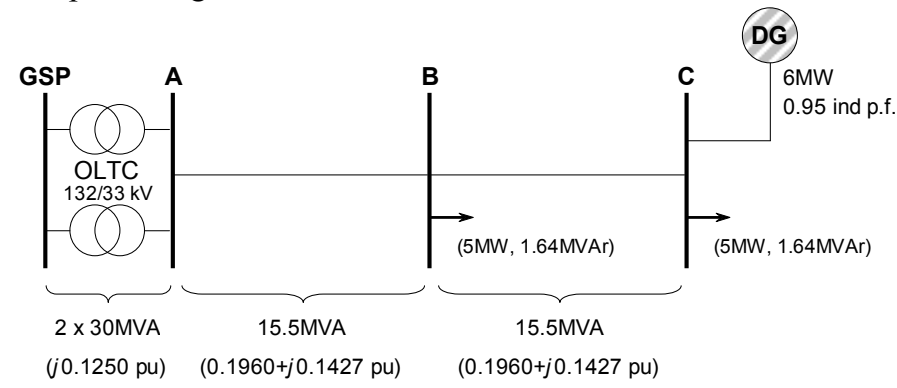

Fig. 1. 33kV 4-bus test feeder (100MVA base) during maximum demand. OLTC set to regulate bus A to $1.00 \mathrm{pu}$.

Assuming an steady output of $6 \mathrm{MW}$ by the DG unit $(0.95$ inductive power factor), and reverse power flow capabilities at the substation, Fig. 3 presents voltage magnitudes and angles using the demand levels adopted in Fig. 2. As expected, it can be observed that the voltage profiles are pushed up due to the power injection from the generator. More importantly, the corresponding phase angle is able to reflect the interaction between loads and generation in the feeder.

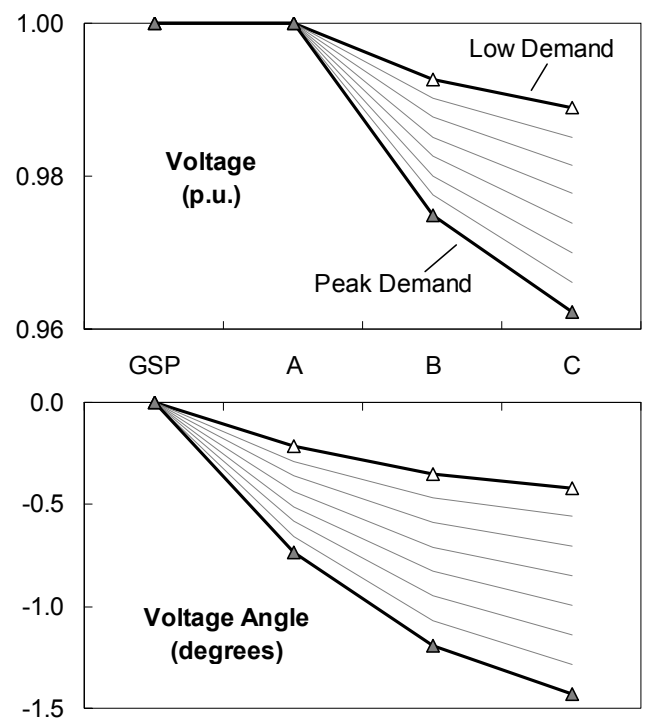

Fig. 2. (Top) Voltage magnitudes and (Bottom) voltage angles at every node of the $33 \mathrm{kV}$ 4-bus test feeder considering different levels of demand and no generation.
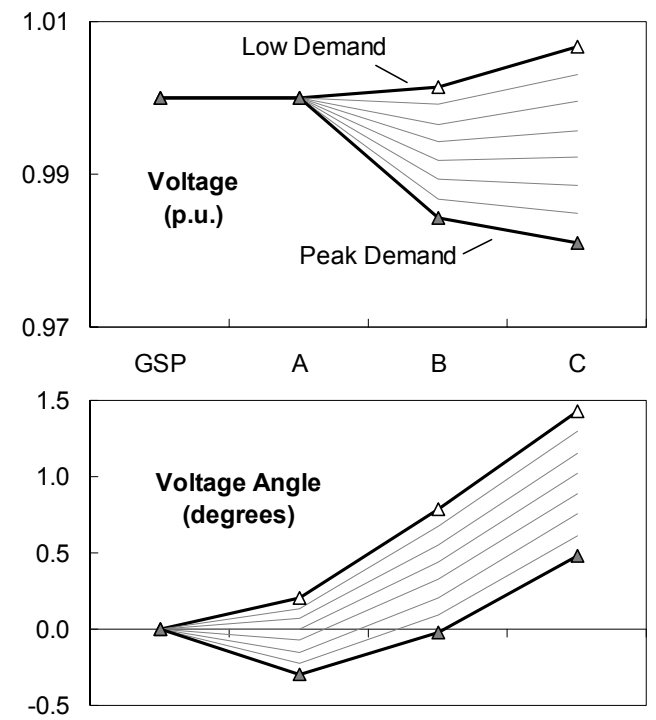

Fig. 3. (Top) Voltage magnitudes and (Bottom) voltage angles at every node of the $33 \mathrm{kV}$ 4-bus test feeder considering different levels of demand and $6 \mathrm{MW}$ of distributed generation.

During peak demand (10MW), part of the power output from the DG plant is locally consumed by the load at bus C, with the reminder exported upstream. The phase angle at bus $\mathrm{C}$ becomes positive. At bus $\mathrm{B}$, the load is served mostly by the GSP but also by the generator. The corresponding angle, while not positive, was pushed up significantly. Finally, the angle at bus A clearly results in a demand-led (i.e., negative) angle, 


\section{Accepted Paper}

although much smaller than that without DG. On the other hand, when the feeder is a net exporter during minimum demand conditions (3MW), all the phase angles become positive.

While voltage magnitudes do show the effects of power injections from DG units (i.e., voltage rise), the spectrum of values are in general not clear enough to adequately distinguish among scenarios (e.g., low demand-no generation vs. higher demand-higher generation), particularly when there is more than one generator connected. This distinction is better achieved by the phase angle. Indeed, the phase angle is a particularly versatile measurement that can be used as proxy to determine when lines or buses are close to their corresponding thermal or voltage limits, as well as any other network constraint such as reverse power flows through grid transformers.

\section{Angle Constraint Active Management (ACAM)}

Connection of renewable generation capacity that is beyond the traditional fit-and-forget approach of a given distribution network can only happen (unless reinforcements are carried out) if the corresponding binding constraints are somehow actively managed. Whether it is a voltage, thermal or reverse power flow issue, measurements have to be made to feed the control mechanism that will in turn determine and perform the best preventive (or corrective) actions. These measurements are traditionally carried out by multiple scalar voltage and current measurements, characterised by nonsynchronised data transmitted once every few seconds. Depending on the scheme, many measuring points (and the corresponding data communications) may be needed.

This work proposes the use of synchrophasor measurements to actively manage wind power generation output in congested distribution networks. This is achieved by applying a fixed angle-based constraint that is determined through network analysis (i.e., a proxy for thermal and voltage limits, avoidance of reverse power flow through grid transformers, etc.) and using minimal communication.

Fig. 4 shows the same test feeder presented previously but with two wind farms connected to it. It is assumed that WF1 has been granted firm connection as no further reinforcements where needed to accommodate it (the $\mathrm{N}-1$ condition for the substation is as restrictive as line A-B). WF2, on the other hand, corresponds to a new potential development. It is important to highlight that given the firm connection agreement between WF1 and the local DNO, its power production will continue to follow its normal pattern, disregarding the effects that a new generator might have on the network. Consequently, any potential congestion must be managed solely by WF2.

For this test feeder, the available fit-and-forget capacity at bus $\mathrm{C}$ is not only limited by that of line $\mathrm{B}-\mathrm{C}$ but also by line A-B (15.5MVA). The corresponding value is obtained by considering minimum demand conditions (3MW) and

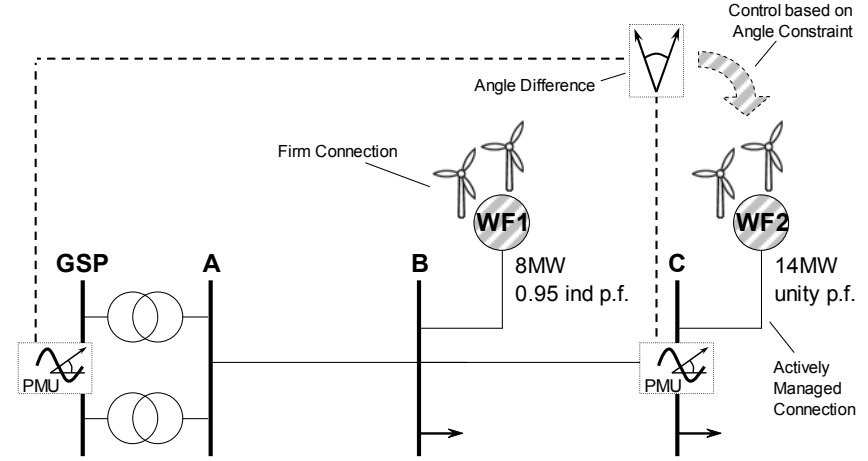

Fig. 4. 33kV 4-bus test feeder with two wind farms (WF1 and WF2), including the Phasor Measurement Units (PMUs) for the proposed Angle Constrained Active Management.

maximum generation of WF1 (8MW). Thus, the fit-and-forget capacity is approximately $10 \mathrm{MW}$. Therefore, the planned $14 \mathrm{MW}$ of the case presented in Fig. 4 will require either network reinforcements or active management of its power output in order to avoid thermal issues during certain critical scenarios.

The proposed implementation of the Angle Constraint Active Management (ACAM) scheme would include two Phasor Measurement Units (PMUs), one at the substation and one at the connection point of the wind farm whose output will be actively managed. The voltage angle difference between these two PMUs will be constantly compared against the fixed angle constraint (found according to the network characteristics - explained below). If the angle difference is above the angle constraint, then the control mechanism is triggered, leading to a reduction of the power output of WF2 in a way that maintains the angle difference within limits, and hence keeps the network secure.

The assessment of when to curtail WF2 and by how much capacity, and, consequently, determining the angle constraint, will depend on the different generation levels of WF1 (combined with the different levels of demand). WF2 will have to 'adapt' to the remaining capacity to transfer its power output. Thus, if for a given load and WF1 generation scenario (say minimum demand-nominal output of WF1), the nominal output of WF2 exceeds the network limits, then it is possible to evaluate the maximum possible injection of WF2 and its corresponding angle. One way of doing so is by exhaustive power flow analyses. Another more sophisticated way is using an optimisation technique such as a tailored ac Optimal Power Flow (OPF) [2].

Fig. 5 shows the phase angles at bus $\mathrm{C}$ resulting from accommodating the nominal capacity of WF2 (14MW) considering low and peak demands combined with five generation levels of WF1 (diagonal-patterned bars). Results also include the phase angles of those scenarios where the full 14MW must be constrained (due to the thermal limit of line A$\mathrm{B}$; grey bars) and those where the maximum injection from WF2 was calculated with the tailored OPF (white bars).

The dash line across all the scenarios corresponds to a phase angle of $3.77^{\circ}$ obtained when $10.2 \mathrm{MW}$ are injected by 


\section{Accepted Paper}

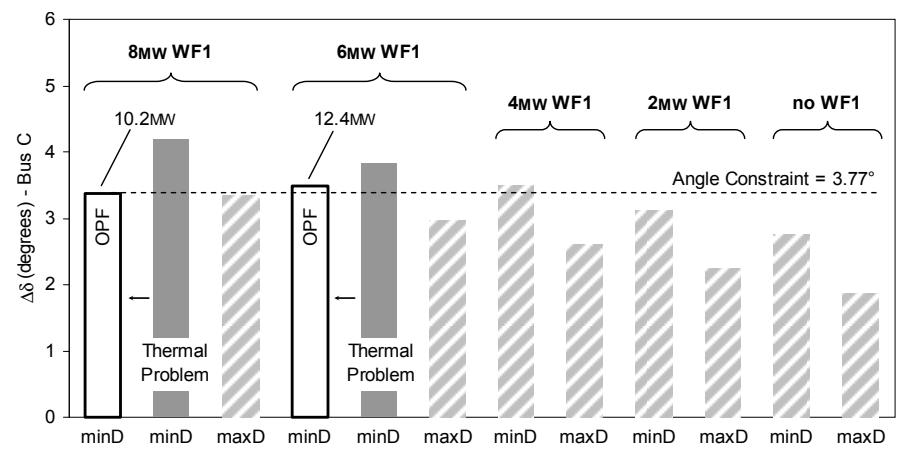

Fig. 5. Phase angles at bus $\mathrm{C}$ resulting from accommodating the nominal capacity of WF2 (14MW) considering low and peak demands combined with five generation levels of WF1.

WF2 during minimum demand and nominal generation of WF1 (i.e., fit-and-forget capacity). Given that this angle is smaller than that found by the OPF solution in the 6MW WF1minimum demand scenario, it becomes the angle constraint for the ACAM scheme as no angle below this value could result in a scenario where thermal or voltage limits are exceeded.

According to the results presented in Fig. 5, the angle constraint will restrict the generation output of not only those scenarios where there is a thermal problem (grey bars) but also the 4MW WF1-nominal WF2 scenario (although marginally). However, in practical terms, the occurrence of curtailment due to the angle constraint will entirely depend on how frequently high volumes of wind power and minimum demand coincide. Consequently, to evaluate the performance of the proposed methodology, the time-varying characteristics of demand and generation need to be taken into account for a given period.

To illustrate this, hourly demand and wind power data for central Scotland in 2003 is adopted [7]. It is assumed that both wind farms, WF1 and WF2, are geographically close, so only one wind profile is required for the area. A generic wind power curve is adopted. An adaptation of the multi-period ac OPF from [2], able to handle multiple demand and generation scenarios, is used to determine the energy curtailed resulting from using the proposed angle constraint technique. Fig. 6 shows the total energy exported and the corresponding capacity factor for WF2 considering: (1) 10.2MW of fit-andforget capacity; and, (2) 14MW of generation capacity where the ACAM scheme is applied (angle constraint equal to $3.77^{\circ}$ ).

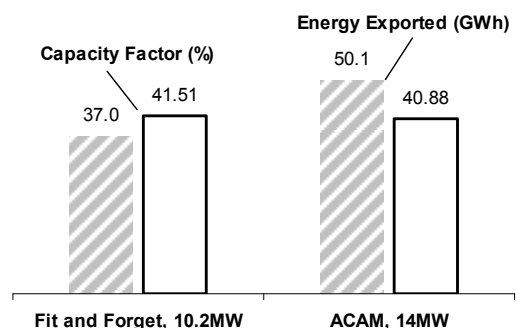

Fig. 6. Energy exported and capacity factor for WF2 considering: (1) $10.2 \mathrm{MW}$ of 'fit and forget' capacity; and, (2) 14MW of generation capacity where the ACAM scheme is applied.

By using the ACAM scheme is indeed possible to connect $14 \mathrm{MW}$, allowing an increase of $37 \%$ in terms of capacity compared to the fit-and-forget case. However, as discussed previously, due to network (thermal) constraints, generation curtailment of WF2 occasionally occurs, particularly during critical scenarios of minimum demand-maximum generation scenarios. The resulting volume of energy exported increases by $35 \%$. From the capacity factor perspective, a metric that gives an idea to wind farm developers about the costeffectiveness of a project, the healthy 0.415 figure of the fitand-forget approach goes down to 0.408 , i.e., a $2 \%$ decrease. Ultimately, although in this case marginal, the perceived loss of revenue will have to be balanced out with the investment required for the extra generation capacity, the corresponding ACAM scheme, and the extra volume of energy exported.

\section{DISCUSSION}

Clearly, the ACAM scheme makes it possible to connect generation capacities beyond the fit-and-forget limit. However, the actual cost-effectiveness of a given wind farm will mainly depend on how frequent its power output is curtailed. Consequently, technical and economic studies for active management schemes require considering the various interactions between local demand and generation, and the corresponding network constraints. The cost of the extra generation capacity that can be connected relative to the overall capacity factor (or, in other words, the loss of revenue), will ultimately define the economic feasibility of an actively managed wind farm development.

It can be argued that thermal constraints could be monitored by simple current measurements that can feed into a control mechanism of an actively managed wind farm, achieving the same performance as the ACAM scheme. In fact, that represents an alternative approach if it is indeed possible to place measurement units at the line or transformer where the thermal constraint is. However, this is not necessarily always the case. In addition, the versatility of the ACAM scheme relies on the fact that it can cater for multiple constraints at the same time, given that the angle constraint acts as proxy. Finally, changes in network topology or infrastructure directly affect the angle difference, making it possible for the ACAM scheme to react accordingly without compromising network security.

\section{CONCLUSIONS}

The innovative use of synchrophasor technology to actively manage wind power generation output in congested distribution networks has been proposed in this work. This is achieved by applying an angle-based constraint that is determined according to the network characteristics (i.e., a proxy for thermal limits, voltage limits, etc.) and using minimal communication. Results have demonstrated that more energy can be exported as opposed to the fit-and-forget approach, although at the expense of smaller capacity factors, whilst keeping the system secure. The economic feasibility of such a scheme will however depend on the cost of the extra generation capacity that can be connected relative to the overall capacity factor (or, in other words, the loss of revenue). 


\section{REFERENCES}

[1] Office of Gas and Electricity Markets (Ofgem), "Electricity distribution price control review - Final proposals - Incentives and Obligations," Dec. 2009.

[2] L. F. Ochoa, C. Dent, and G. P. Harrison, "Distribution network capacity assessment: Variable DG and active networks," IEEE Trans. on Power Systems, vol. 25, no. 1, pp. 87-95, Feb. 2010.

[3] L. F. Ochoa and G. P. Harrison, "Minimizing energy losses: Optimal accommodation and smart operation of renewable distributed generation," IEEE Trans. on Power Systems, In Press. Available online, DOI:10.1109/TPWRS.2010.2049036.

[4] L. F. Ochoa, L. C. Cradden, and G. P. Harrison, "Demonstrating the capacity benefits of dynamic ratings in smarter distribution networks," in Proc. 2010 IEEE/PES Conference on Innovative Smart Grid Technologies, pp. 6.

[5] IEEE Standard for synchrophasors for power systems, IEEE Std C37.118-2005, 2006.

[6] D. H. Wilson, "Wide-area measurement and control for dynamic stability," in Proc. 2007 IEEE/PES General Meeting, pp. 5.

[7] T. Boehme, J. Taylor, A. R. Wallace, and J. W. Bialek, "Matching renewable electricity generation with demand," Scottish Executive, Edinburgh, Feb. 2006.

\section{BIOGRAPHIES}

Luis F. Ochoa ( $\left.\mathrm{S}^{\prime} 01-\mathrm{M}^{\prime} 07\right)$ is an associate at Psymetrix and a Research Fellow in the School of Engineering, University of Edinburgh, U.K. He obtained his BEng degree from UNI, Lima, Peru, in 2000, and the MSc and $\mathrm{PhD}$ degrees from UNESP, Ilha Solteira, Brazil, in 2003 and 2006, respectively.

His current research interests include network integration of distributed energy resources and distribution system analysis. Dr. Ochoa is also a member of the IET.

Douglas H. Wilson graduated BEng (Hons) in 1991 and $\mathrm{PhD}$ in 1997 from the University of Edinburgh, Scotland, UK, and MSc in 2006 from the University of Edinburgh. He is currently the Chief Technical Officer for Psymetrix Limited, working on systems and applications for real-time power system stability management. 